\title{
COLLOQUIUM PARTICIPANTS
}

V.I. Abramenko, Crimean Astrophysical Obs., 334413 Nauchny, Crimea, Ukraine

Guoxiang Ai, Beijing Astronomical Observatory, Chinese Academy of Sciences Beijing 100080, P. R. China

Maki Akioka, Hiraiso Solar Terrestrial Research Center Communications Research Lab., Ministry of Post and Telecommunications, Isozaki Nakaminato, Ibaraki 311-12, Japan

Stepanov Alexander, Crimean Astrophysical Obs., Ukraine, 334247, Crimea, Yalta, Ratziveli, RT 22

Pavel Ambroz, Astronomical Institute of the Czechoslovak, Academy of Sciences, Observatory Ondrejov, CS-251 65, Ondrejov, Czechoslovakia

Hanslmeier Arnold, Institut fur Astronomie Univ. -Platz 5, A-8010 GRAZ, Austria

J.-L. Astor, CNES-Department Publications, Centre Spatial de Toulouse-18, Avenue Edouard Belin-31055 Toulouse Cedex, France

Dollfus Audouin, Observatoire de Paris, 92195-Meudon Cedex, France

Xingming Bao, Urumqi Astronomical Station, Xinjiang 830011, P. R. China

A. Bhatnagar, Udaipur Solar Obs. 11, Vidya Marg, Udaipur-313001, India

V.M. Bogod, Special Astrophysical Obs. of the Russian Academy of Science, St. Petersburg Branch, St. Petersburg, 196140, Russia

Javier Trujillo Bueno, Instituto de Astrofisica de Canarias E-38200 La Laguna, Tenerife, Spain

V. Bumba, Czechoslovak Academy of Sc., Astronomical Institute, 25165 Ondrejov, Czechoslovakia

V.A. Burov, IZMIRAN, 142092 Troitsk, Moscow Region, Russia

Suresh Chandra, 14 Hirapuri Colony New Construction, University Campus, Gorakhpur 273009, India

Jimin Chen, Beijing Astronomical Observatory, Chinese Academy of Sciences, Beijing 100080, P. R. China 
Zhencheng Chen, Beijing Astronomical Observatory Chinese Academy of Sciences, Beijing 100080, P. R. China

Tzihong Chiveh, Physics Dept., National Central Univ., Chung-Li, Taiwan

Choe Won Chol, Pyongyang Astronomical Obs. Academy of Sciences of DPR of Korea

Dean-Yi Chou, Physics Dept., Tsing Hua University, Hsinchu, 30043, Taiwan

Joseph Darik, NASA-Goddard Space Flt. Cen., Code 682 Greenbelt MD 20771, USA

Prasad C. Debi, Inter-University Center for Astronomy and Astrophysics Post Bag 4, Ganeshkhind, Pune 411007 , India

D. Deming, NASA-Goddard Space Flight Center, Greenbelt MD 20771, Maryland, USA

X. H. Deng, USTC, Center for Astrophysics, Hefei 230026, P. R. China

Franz-L. Deubner, Institut fur Astronomie and Astrophysik der Universitat Wurzburg, 8700 Wurzburg-Am Hubland, Germany

Mingde Ding, Dept. of Astronomy, Nanjing University, Nanjing 210008, P. R. China

Landi Degl'innocenti, Dipartimento Di Astronomia, Universita Di Firenze Egidio, Largo E. Fermi, 5-50125 Firenze, Italy

Shinzo Enome, Nobeyama Radio Observatory, Minamisaku, Nagano 384-13, Japan

L. V. Ermakova, Russia

Cheng Fang, Department of Astronomy, Nanjing University, Nanjing 210008, P. R. China

Bernhard Fleck, Istitut fuer Astronomie and Astrophysik, Universitaet Wuerzburg AM Hubland, D 8700 Wuerzburg, F. R. Germany

T. Forbes, Space Science Center EOS, Science Engin. Res., BIdg Univ. of New Hampshire, Durham NH 03857, USA

Qijun Fu, Beijing Astronomical Observatory, Chinese Academy of Sciences, Beijing 100080 , P. R. China

V. Gaizauskas, National Research Council of Canada, 100 Sussex Drive, Canada 
G.B. Gelfreikh, Special Astrophysical Obs. of the Russian, Academy of Science, St., Petersburg Branch, St., Petersburg, 196140, Russia

A.A. Golovko, SibIZMIR, P. O. Box 4, Irkutsk 664033, Russia

Philip Goode, Professor of Physics, New Jersey Institute of Technology, Newark, New Jersey 07102, USA

S.I. Gopasyuk, Crimean Astrophysical Obs., 334413 Nauchny, Crimea, Ukraine

V.M. Grigoryev, SibIZMIR, P. O. Box 4, Irkutsk 664033, Russia

X. M. Gu, Yunnan Astronomical Observatory, Chinese Academy of Sciences, Kunming 650011, P. R. China

M. Hagyard, NASA Marshall SFC, Code ES 52, Huntsville, AL 35812, USA

John W. Harvey, National Solar Obs. 950 N. Cherry Ave. P. O. Box 26732 Tucson, Arizona 85726-6732, USA

Karen L. Harvey, Solar Physics Research Corporation, 4720 Calle Desecada, Tucson, AZ 85718, USA

Vladimir Hayrapetyan, 378433, Byurakan Obs., Armenian Academy of Sc., ARMENIA

Jean-Claude Henoux, Ministere De L'Education Nationale Observatoire de Paris, Section D'astrophysique, 92195 Meudon, France

A. Hofmann, Astrophysikalisches Institut Potsdam, Sonnenobservatorium Einsteintum, 0-1561 Potsdam, Telegrafenberg

Robert F. Howard, National Solar Obs., P. O. Box 26732, Tucson, AZ 85726, USA

Ju Hu, Dept. of Astronomy, Nanjing University, Nanjing 210008, P. R. China

Guangli Huang, Purple Nountain Observatory, Nanjing 210008, P. R. China

Kiyoshi Ichimoto, National Astronomical Obs. of Japan, Mitaka, Tokyo 181, Japan

Tohmura Ichiroh, Osaka Prefectural College of Technology Saiwai-cho, Neyagawa, Osaka 572, Japan

B.A. Ioshpa, IZMIRAN, 142092 Troitsk, Moscow Region, Russia

V.N. Ishkov, IZMIRAN, 142092 Troitsk Moscow Region Russia 
Li Sang Jae, Pyongyang Astronomical Obs. Academy of Sciences of DPR of Korea

Stuart Jefferies, National Optical Astronomy Observatories, P. O. Box 26732, Tucson, AZ 85726-6732, USA

Eberhart Jensen, Institute of Theoretical Astrophysics, University of Oslo, Blindem, P. O. Box 1029, Oslo, Norway

Jiehai Jin, Purple Mountain Observatory, Academia Sinica, Nanjing 210008, P. R. China

Shu-ping Jin, Department 7, Chinese Technology Univ., Hefei 230026, P. R. China

Sanchez Almeida Jorge, Instituto de Astrofisica de Canarias, E-38200 La Laguna, Tenerife, Spain

R. Kariyappa, Indian Institute of Astrophysics, Koramangala Bangalore-560034, India

Singo Kawakami, Osaka Meseum of Sc., 4-2-1 Nakanoshima, Klta-ku, Osaka 530, Japan

Christoph U. Keller, Institute of Astronomy, ETH-Zeutrum, CH-8092 Zurich, Switzerland

Takeo Kosugi, National Astronomical Obs., Mitaka, Tokyo 181, Japan

Valerij N. Krivodubskij, Kiev University, Astronomical Obs., Observatoma Street 3, Kiev-53 25403, Ukraine

L. Krivsky, Astronomical Institute of the, Czechoslovak Academy of Sciences, 251 65 Observatory, Ondrejov, Czechoslovakia

A. Kruger, Astrophysikalisches Institut, Potsdam, Rosa-Luxemburg-Str. 17a, D-0-1591 Postdam, Germany

G.V. Kuklin, SiblZMIR, P. O. Box 4, Irkutsk 664033, Russia

M. Kundu, Astronomy Program, Univ. of Maryland, College Park, Mo 20742, USA

H. Kurokawa, Hida Observatory, Kyoto Univ., Kamitakara 506-13, Gifu, Japan

John K. Lawrence, Dept. of Physics and Astronomy, San Femando Obs., California State University, Northridge Northridge, CA 91330, USA 
Jeongwoo W. Lee, Solar Astronomy 264-33, Califomia Institute of Technology, Pasadena CA 91125 , USA

L. C. Lee, Geophysical Institute, Univ. of Alaska, Fairbanks, Alaska 99775-0800, USA

Chunsheng Li, Department of Astronomy, Nanjing University, Nanjing 210008,P. R. China

Wei Li, Beijing Astronomical Observatory, Chinese Academy of Sciences, Beijing 100080, P. R. China

Xing Li, Dept. of Earth and Space Sciences, USTC, Hefei 230026, P. R. China

J. Lin, Yunnan Astronomical Obs., Chinese Academy of Sciences, Kunming 650011, P. R. China

Yuanzhang Lin, Beijing Astronomical Observatory, Chinese Academy of Sciences, Beijing 100080, P. R. China

Yang Liu, Beijing Astronomical Observatory, Chinese Academy of Sciences, Beijing 100080, P. R. China

Edward Lu, High Altitude Obs., P. O. Box 3000, Boulder, CO 80307, USA

Baorong Luo, Yunnan Astronomical Obs., Chinese Academy of Sc., Kunming 650011, P. R. China

G. Lustig, Institut fur Astronomie, Universitatsplatz 5, A-8010 GRAZ/Austria/Europe

V.I. Makarov, Kislovodsk Station of the Pulkovo Observatory, Kislovodsk 357741, Russia

Mitsugu Makita, Kwasan Observatory, Kyoto University, Yamashina, Kyoto 607, Japan

Robillot Jean Maurice, Observatoire de Bordeaux 33270 Floirac, France

Nicole Mein, Observatoire de Paris, Section de MEUDON, 5 Place Janssen F. 92195, MEUDON Pae CEDEX

Pierre Mein, Observatoire de Paris, Section de MEUDON, 5 Place Janssen F. 92195, MEUDON Pae CEDEX

Changrong Ming, Beijing Astronomical Observatory, Chinese Academy of Sciences, Beijing 100080, P. R. China 
E.I. Mogilevsky, IZMIRAN, 142092 Troitsk, Moscow Region, Russia

Yoshinari Nakagawa, Chiba Institute of Technology, Narashino 275, Japan

A. Nezis, Kiepenheuer-Institut, Schoneckstr. 6, D-7800, Freiburg-W., Germany

Luigi Nocera, Istituto Di Fisica Atomica E Molecolare-CNR Via Giardino, I-56127 PISA, Italy

V.N. Obridko, IZMIRAN, 142092 Troitsk, Moscow Region, Russia

K. Petrovay, Eotvos University, Department of Astronomy Budapest, ludovika ter 2, H-1083 Hungary

\section{A.A. Pevtsov, Russia}

Valentin Martinez, Instituto de Astrofisica de Canarias, Pillet E-38200 La Laguna, Tenerife, Spain

G.A. Porfir'eva, Hemberg Astronomical Institute, University Prospect, 13, Moscow, 119899, Russia

Z.Q. Qu, Yunnan Astronomical Obs., Chinese Academy of Sciences, Kunming 650011 , P. R. China

Probhas Raychauduri, Dept. of Applied Mathematics, Calcutta University, Calcutta-700009, India

Bocchia Romeo, Observatoire de Bordeaux, 33270 Floirac, France

Robert J. Rutten, Sterrekundig Instituut Postbus 80 000, NL-3508 TA Utrecht, Netherlands

Vladimir Ruzdjak, Hvar Observatory, Faculty of Geodesy, Kaciceva 26, 41000 Zagreb, Croatia

M. Ryutova, Institute of Nuclear Physics, 630090 Novosibirsk, Russia

Metod Saniga, Astronomical Institute, Slovak Academy of Sciences, CS-059 60 Tatranska Lomnica, Czechoslovakia

Taro Sakao, National Astronomical Obs., 2-21-1, Mitaka, Tokyo 181, Japan

Takashi Sakurai, National Astronomical Obs. of Japan, 2-21-1 Ohsawa, Mitaka, Tokyo 181, Japan 
Norbert Seehafer, AG Nichtlineare Dynamik, Universitat Potsdam, Am Neuen Palais, Gebaude 5, 0-1571 Potsdam, Germany

V. D. Selivanov, Russia

Zhongxian Shi, Beijing Astronomical Observatory, Chinese Academy of Sciences, Beijing 100080, P. R. China

Kiyoto Shibasaki, Nobeyama Radio Observatory, Minamimaki, Minamisaku, Nagano 384-13, Japan

K. Shibata, National Astronomical Obs., Solar Physics Division, Mitaka, Tokyo 181, Japan

George Simon, AFGL-PHS, National Solar Observatory, Sunspot NM 88349, USA Jagdev Singh, Indian Institute of Astrophysics, Bangalore 560034, India

R. N. Singh, Dept. of Applied Physics, Institute of Technology, Banaras Hindu University Varanasi-221005, India

V.I. Skomorovsky, Russia

G. Smolkov, SIBIZMIR, Irkutsk 33, P. O. Box 4026, 664033, Russia

Michal Sobotka, Instituto De Astrofisica, 38200 La Laguna, Tenerife, Spain

S.K. Solanki, Inst. of Astronomy, ETH-Zurich, CH-8092 Zurich, Switzerland

Dirk Soltau, Kiepenheuer-Instutut fur Sonnenphysik, Schoneckstra B e 6, D-7800 Freiburg, Germany

Weihong Song, Physics Department, Yunnan Normal University, Kunming, P. R. China

Raphael Steinitz, Physics Dept., Ben Gurion University, 84105 Beer Sheva, ISRAEL

R.S. Steinolfson, Southwest Research Institute, 6220 Culebra Road San Antonio, TX 78230-5166, USA

J. Stenflo, Institute of Astronomy, ETH-Zentrum, CH-8092 Zurich, Switzerland

G. Sudakova, Krasnoyarsk State University, Post Box 8630, Academgorodok 36, Krasnoyarsk 660036, Russia

Kai Sun, Geophysics Department, Peking University, Beijing 100871, P. R. China 
Yuhua Tang, Dept. of Astronomy, Nanjing University, Nanjing 210008, P. R. China

John H. Thomas, Mechanical Engineering Department, University of Rochester, Hopeman 233, River Campus, Rochester, NY 14627, USA

A. Title, Solar Phy., Dept. 9130/256 Lockheed Research Labs., 3251 Hanover St. Palo Alto, CA 94303, USA

Eduardo Mendoza Torres, Special Astrophysical Obs., Russian Academy of Sciences, 37-2, St. Petersburg, 134003, Russia

Chang-Hua Tsao, Bartol Research Institute, Univ. of Delaware, Newark, DE 19711, USA

Saku Tsuneta, Institute of Astronomy, Univ. of Tokyo 2-21-1 Osawa Mitaka, Tokyo 181, Japan

Lidia van Driel-Gesztelyi, Rijksuniversiteit te Utrecht, Sterrekundig Insituut, Postbus 80 000, 3508 TA Utrecht, The Netherlands

Arthur H. Vaughan, Jet Propulsion Laboratory, Mail Code 179-225, 4800 Oak Grove Drive, Pasadena, Califomia 91109, USA

Haimin Wang, Califomia Institute of Technology, Solar Astronomy 264-33, Pasadena, CA 91125, USA

Huaning Wang, Beijing Astronomical Observatory, Chinese Academy of Sciences, Beijing 100080 , P. R. China

Jia-Long Wang, Beijing Astronomical Observatory, Chinese Academy of Sciences, Beijing 100080, P. R. China

Jingxiu Wang, Beijing Astronomical Observatory, Chinese Academy of Sciences, Beijing 100080, P. R. China

Y.-M. Wang, Department of the Navy, Naval Research Laboratory, Washington D.C. 20375-5000, USA

Peter R. Wilson, School of Mathematics, University of Sydney, NSW 2006, Australia

Linxiang Wu, Dept. of Geophysics, Peking University, Beijing 100871, P. R. China

S.T. Wu, Center for Space Plasma and Aeronomic Research, The University of Alabama in Huntsville, Huntsville, Alabama 35899, USA 
Zhiguo Xia, Yunnan Astronomical Observatory, Chinese Academy of Sciences, Kunming 650011, P. R. China

Aoao Xu, Dept. of Astronomy, Nanjing University, Nanjing 210008, P. R. China

J.Y. Xuan, Yunnan Astronomical Observatory, Chinese Academy of Sciences, Kunming 650011, P. R. China

Yihua Yan, Beijing Astronomical Observatory, Chinese Academy of Sciences, Beijing 100080, P. R. China

Shi-hui Ye, Purple Mountain Observatory, Academia Sinica, Nanjing 210008, P. R. China

Bairong Zhang, Yunnan Astronomical Observatory, Chinese Academy of Sciences, Kunming 650011, P. R. China

Caicheng Zhang, Beijing Astronomical Observatory, Chinese Academy of Sciences, Beijing 100080 , P. R. China

Guiqing Zhang, Beijing Astronomical Observatory, Chinese Academy of Sciences, Beijing 100080, P. R. China

Heng Zhang, Yunnan Astronomical Observatory, Chinese Academy of Sciences, Kunming 650011, P. R. China

Hongqi Zhang, Beijing Astronomical Observatory, Chinese Academy of Sciences, Beijing 100080, P. R. China

Xu Zhang, Institute of Mechanics, Beijing 100080, P. R. China

Renyang Zhao, Beijing Astronomical Observatory, Chinese Academy of Sciences, Beijing 100080, P. R. China

Daoqi Zhou, Dept. of Geophysics, Peking University, Beijing 100871, P. R. China

H. Zirin, Solar Astronomy 264-33, California Institute of Technology, Pasadena CA 91125, USA

Cornelis Zwaan, Sterrekundig Instituut Postbus 80 000, NL-3508TA Utrecht, The Netherlands 